OPEN ACCESS

Edited by:

Petia Kovatcheva-Datchary, University of Würzburg, Germany

Reviewed by: Filipe De Vadder, Université de Lyon, France

Eduardo Garcia-Fuentes, Biomedical Research Institute of Malaga, Spain

*Correspondence: Gabriella Peroni gabriella.peroni01@universitadipavia.it

Specialty section: This article was submitted to

Gut Endocrinology, a section of the journal Frontiers in Endocrinology

Received: 01 February 2021 Accepted: 22 April 2021

Published: 14 May 2021

Citation:

Rondanelli M, Gasparri C, Peroni G, Faliva MA, Naso M, Perna S, Bazire $P$,

Sajoux I, Maugeri R and Rigon C (2021) The Potential Roles of Very Low Calorie, Very Low Calorie Ketogenic Diets and Very Low Carbohydrate Diets on the Gut Microbiota Composition. Front. Endocrinol. 12:662591. doi: 10.3389/fendo.2021.662591

\section{The Potential Roles of Very Low Calorie, Very Low Calorie Ketogenic Diets and Very Low Carbohydrate Diets on the Gut Microbiota Composition}

\author{
Mariangela Rondanelli ${ }^{1,2}$, Clara Gasparri ${ }^{3}$, Gabriella Peroni ${ }^{3 *}$, Milena Anna Faliva ${ }^{3}$, \\ Maurizio Naso ${ }^{3}$, Simone Perna ${ }^{4}$, Philip Bazire ${ }^{5}$, Ignacio Sajoux ${ }^{6}$, Roberto Maugeri ${ }^{7}$ \\ and Chiara Rigon ${ }^{3}$
}

\begin{abstract}
${ }^{1}$ IRCCS Mondino Foundation, Pavia, Italy, ${ }^{2}$ Department of Public Health, Experimental and Forensic Medicine, Unit of Human and Clinical Nutrition, University of Pavia, Pavia, Italy, ${ }^{3}$ Endocrinology and Nutrition Unit, Azienda di Servizi alla Persona "Istituto Santa Margherita", University of Pavia, Pavia, Italy, ${ }^{4}$ Department of Biology, College of Science, University of Bahrain, Sakhir, Bahrain, ${ }^{5}$ PronoKal, London, United Kingdom, ${ }^{6}$ PronoKal Group, Barcelona, Spain, ${ }^{7}$ PronoKal Group, Savigliano, Italy
\end{abstract}

Several studies have described a strong correlation between diet, weight loss, and gut microbiota composition. The aim of this review was to evaluate the potential effects of energy-restricted diets, namely very low calorie diets (VLCDs), very low calorie ketogenic diets (VLCKDs), and very low carbohydrate diets (VLCarbDs), on the composition of the gut microbiota in humans. We performed a literature search using the following terms (with their abbreviations or acronyms): "very low calorie diet", "very low calorie ketogenic diet", "very low carbohydrate diet", and "gut microbiota". Our search strategy retrieved nine eligible studies. Overall, VLCDs and VLCarbDs affected the Bacteroidetes to Firmicutes ratio in obese patients, leading to a reduction in short-chain fatty acid production by fecal microbiota associated with Clostridial cluster XIVa. This reduction particularly affected Roseburia and Eubacterium rectale, the two most abundant butyrate-producing bacteria in human feces. VLCKDs preserved the core fecal microbiome, but altered the composition of fecal microbial populations in relation to the plasma metabolome and fecal bile acid composition. In particular, VLCKD-induced weight loss resulted in a reduction in E. rectale and Roseburia, an increase in Christensenellaceae and Akkermansia while not all studies show a decrease in Faecalibacterium prausnitzii. Although very few studies have analyzed the effects of VLCarbDs and VLCDs on gut microbiota, significant diet-induced changes in fecal microbiota composition have been observed. Further studies are needed.

Keywords: very low carbohydrate diet (VLCarbD), very low calorie diet (VLCD), obesity, microbiota, very low calorie ketogenic diet (VLCKD), gut microbiota 


\section{INTRODUCTION}

The gut microbiota has been recognized as a key factor driving metabolic diseases such as diabetes, atherosclerotic cardiovascular disease, non-alcoholic fatty-liver disease, Alzheimer's disease, and certain obesity-related cancers (1). It also plays an important role as a separate endocrine organ that maintains host energy homeostasis and stimulates host immunity through molecular crosstalk (2-4). Shifts in gut microbial composition due to extrinsic factors can significantly disrupt the symbiotic relationship between gut bacteria and the host, favoring the development of metabolic diseases.

Microbes in the human gut produce a wide range of metabolites, most of which are chemically similar to metabolites produced by host cells (e.g. nitric oxide, gamma-aminobutyric acid, serotonin, short-chain fatty acids [SCFAs], and indoles). Other metabolites, however, such as the bile acids, result from the chemical transformation of host molecules by microbes. Due to their similarity, these molecules are all recognized by host cells, and may act on specific receptors or trigger the release of other hormonal signals, such as the gut peptides glucagon-like peptide1 and peptide YY, which both act on energy metabolism. Translocation of lipopolysaccharides (LPS) into the bloodstream can trigger low-grade inflammation affecting the liver and adipose tissue and altering muscle metabolism; it is an important hallmark of obesity, diabetes, and related disorders. In addition, those endotoxins can disrupt appetite regulation by altering the activity of the enteric nervous system and the gut-brain axis via the vagus nerve (4). The most common organisms in the human gut are members of the gram-positive Firmicutes phylum, which includes the genera Eubacterium, Clostridium, Ruminococcus, Butyrivibrio, and Lactobacillus; the gram-negative Bacteroidetes phylum, with the genera Bacteroides, Prevotella, and Porphyromonas; and the less abundant Proteobacteria, with the genus Enterobacteria (e.g. Escherichia coli); Verrucomicrobiaceae, with Akkermansia municiphila; and Actinobacteria, with the genus Bifidobacterium (5-7). The human gut contains three main enterotypes dominated by Ruminococcus, Bacteroides, and Prevotella. Each enterotype uses a different energy pathway, and it has been hypothesized that the predominance of one genus over another is determined by the host's diet (8). In addition, the composition of gut microbiota may be altered by certain diseases; it has been shown, for example, that the gut microbiota of people with diabetes has a different composition to that of healthy individuals (9). Diabetes and obesity are both characterized by low-grade inflammation of unknown molecular origin. A study by Cani et al. (10) showed that metabolic endotoxemia influences inflammatory tone, weight gain, and diabetes, and that high-fat nutrition modulates gut microbiota and plasma concentrations of LPS. Changes observed in the gut microbiota following antibiotic treatment suggest that these microorganisms might be involved in modulating metabolic endotoxemia, low-grade inflammation, obesity, and type 2 diabetes. This would also provide an explanation for some of the mechanisms involved (11).

Very low calorie diets (VLCDs) (high-protein, lowcarbohydrate [ $\leq 30 \%$ ] diets with a maximum intake of $800 \mathrm{kcal} /$ day) and very low calorie ketogenic diets (VLCKDs) $(<800 \mathrm{kcal} /$ day, carbohydrates $<70 \mathrm{~g} /$ day) are used in interventions designed to reduce body weight and improve fasting blood glucose, insulin, and lipid levels (12). VLCKDs induce ketosis, which is a metabolic state characterized by an increased concentration of ketones (acetoacetate, 3- $\beta$-hydroxybutyrate, and acetone) in the blood due to raised levels of fatty acid breakdown and ketogenic enzyme activity (13). The permitted daily intake is $70-100 \mathrm{~g}$ protein (or 0.8 $1.5 \mathrm{~g}$ protein $/ \mathrm{kg}$ of ideal body weight), $<50 \mathrm{~g}$ of carbohydrates from vegetables, and $10 \mathrm{~g}$ of olive oil (14). VLCKDs involve the complete replacement of regular meals with food or formulations used under medical supervision for weight loss in individuals with a body mass index $(\mathrm{BMI})>30$ (or $>27$ if the person has obesity-related comorbidities) and in those requiring rapid weight loss (15). VLCDs are typically used as part of an intervention combining medical supervision and lifestyle changes. Under these conditions, they are generally considered safe and effective (14-16) and are gaining traction as a means of achieving rapid weight loss.

The aim of this review was to examine the potential effects of very low calorie diets (VLCDs), very low calorie ketogenic diets (VLCKDs) and very low carbohydrate diets (VLCarbDs) on the composition of the gut microbiota. To date, the studies on this topic in literature are scarce. Since gut microbiota is involved with the quantity and quality of nutrients extracted from the diets, with direct implications for obesity and related disorders, it's interesting to investigate how VLCDs and VLCKDs affect the composition of the gut microbiota, and whether these modifications may be effective against obesity, diabetes, and related disorders.

\section{MATERIALS AND METHODS}

The present systematic review was performed following the steps by Egger et al. as follows (17): (1) A working group was configured as follows: three operators skilled in clinical nutrition, of whom one acting as a methodological operator and two participating as clinical operators. (2) The revision question on the basis of considerations made in the abstract was formulated as follows: "the state of the art of the effects of energy-restricted diets, namely very low calorie diet (VLCD), very low calorie ketogenic diet (VLCKD), and very low carbohydrate diet (VLCarbD), on the composition of the gut microbiota in humans." (3) Relevant studies were identified as follows: a research strategy was planned, on PubMed, Cochrane Central Register of Controlled Trials (CENTRAL) and Web of Science as follows: (a) definition of the key words (Very low carbohydrate diet (VLCarbD), Very low calorie diet (VLCD), obesity, microbiota, Very low calorie ketogenic diet (VLCKD), gut microbiota), allowing the definition of the interest field of the documents to be searched, grouped in quotation marks (“..."), and used separately or in combination; (b) use of the Boolean AND operator that allows the establishment of logical relations among concepts; (c) research modalities: advanced search; (d) limits: time limits: papers published in the last 15 years; languages: English; (e) manual search performed by the senior 
researchers experienced in clinical nutrition through revision of reviews and individual articles on the effects of VLCD, VLCKD, and VLCarbD, on the composition of the gut microbiota in humans in journals qualified in the Index Medicus. (4) The analysis was carried out in the form of a systematic review of the reports.

\section{RESULTS}

The research has been carried out based on the following keywords: "very low calorie diet" OR "VLCD" OR "very low calorie ketogenic diet" OR "VLCKD" AND "gut microbiota"; This search retrieved six eligible studies, shown in Table 1. Three, of the six studies, are randomized controlled trials (1820 ), one prospective cohort study (21), one controlled parallel design trial (22) end one randomized, single blind, parallel design trial (23). The second research has been based on: "very low carbohydrate diets" OR "VLCarbDs" AND "gut microbiota".
This search strategy retrieved three eligible studies, all randomized controlled trial, summarized in Table 2 (24-26).

Figure 1 shows the study selection process.

\section{DISCUSSION}

The human gut contains a complex community of microbes that have a pivotal role in human health. It is estimated to contain around 1000 bacterial species and 100 times more genes than the human genome (27). This community of microbes is often referred to as a "hidden metabolic organ" because of its enormous influence on host metabolism, physiology, nutrition, and immune function (28).

Any dietary changes (e.g., FODMAPs, gluten free or VLCDs), despite showing beneficial effects, can affect microbiota composition, especially when protracted for a long time (29).

During a weight loss program, the relative abundance of Bacteroidetes increased and the abundance of Firmicutes

TABLE 1 | Studies on the effects of VLCDs on gut microbiota.

\begin{tabular}{|c|c|c|c|c|c|}
\hline AUTHOR, YEAR & $\begin{array}{l}\text { TYPE OF } \\
\text { STUDY }\end{array}$ & TYPE OF DIET & SAMPLE & DURATION & FINDINGS \\
\hline Simoes, 2014 (18) & $\begin{array}{l}\text { Randomized } \\
\text { trial }\end{array}$ & $\begin{array}{l}\text { VLCD (VLED) } \\
800 \text { kcal, CHO } 67 \mathrm{~g}, \\
\text { PROT } 90 \mathrm{~g}, \text { FAT } 9.5 \mathrm{~g}\end{array}$ & 16 obese patients & 12 months & $\begin{array}{l}\text { Changes in fecal microbial numbers in obese individuals were } \\
\text { primarily affected by dietary } \\
\text { intake rather than by weight change. } \\
\text { Bifidobacteria and Lactobacillus decreased after the VLED, but } \\
\text { the change was transient. }\end{array}$ \\
\hline $\begin{array}{l}\text { Sandrine, } 2016 \\
\text { (19) }\end{array}$ & $\begin{array}{l}\text { Randomized } \\
\text { trial }\end{array}$ & $\begin{array}{l}\text { VLCKD (VLCD) } \\
(800 \mathrm{Kcal}, \mathrm{CHO}<30 \%)\end{array}$ & 16 obese patients & 52 weeks & $\begin{array}{l}\text { Reduction in the Bacteroidetes : Firmicutes ratio was related to } \\
\text { metabolic syndrome rather than BMI. VLCD was associated with } \\
\text { a reduction in Roseburia and an increase in Akkermansia. } \\
\text { Bifidobacteria numbers did not fall, possibly due to the presence } \\
\text { of inulin. }\end{array}$ \\
\hline $\begin{array}{l}\text { Basciani, } 2020 \\
(20)\end{array}$ & $\begin{array}{l}\text { Randomized } \\
\text { controlled } \\
\text { trial }\end{array}$ & $\begin{array}{l}\text { VLCKD (VLCD) } \\
90 \mathrm{~g} \text { protein } \\
26 \mathrm{~g} \text { carbohydrates } \\
15 \mathrm{~g} \text { lipids }\end{array}$ & $\begin{array}{l}48 \text { obese patients } \\
\text { divided in three group: } \\
\text { 1- } \text { WPG } \\
\text { 2- } \text { VPG } \\
\quad \text { APG }\end{array}$ & 45 days & $\begin{array}{l}\text { After diet, the relative abundance of Firmicutes was significantly } \\
\text { decreased while Bacteroidetes increased proportionally with the } \\
\text { only exception in the VPG in which the increase in Bacteroidetes } \\
\text { not reached statistical significance suggesting that the origin of } \\
\text { proteins may influence the microbiota change }\end{array}$ \\
\hline Aleman, 2018 (21) & $\begin{array}{l}\text { Prospective } \\
\text { cohort study }\end{array}$ & $\begin{array}{l}\text { VLCKD (VLCD) } \\
54 \% \text { PROT } \\
26 \% \text { CHO } \\
20 \% \text { FAT }\end{array}$ & $\begin{array}{l}1-10 \text { obese } \\
\text { postmenopausal } \\
\text { women }\end{array}$ & 46 days & $\begin{array}{l}\text { Roseburia decreased and Christensenellenaceae increased after } \\
\text { VLCKD. No significant changes were observed in the } \\
\text { Bacteroidetes : Firmicutes ratio. During ketosis, } \beta \text { - } \\
\text { hydroxybutyrate production was negatively correlated with } \\
\text { F. prausnitzii and Roseburia contrasting with the results of other } \\
\text { studies cited above. The question remains to be explored. }\end{array}$ \\
\hline $\begin{array}{l}\text { Carolina } \\
\text { Guitérrez-Repiso, } \\
\mathbf{2 0 2 1}(22)\end{array}$ & $\begin{array}{l}\text { Controlled } \\
\text { parallel } \\
\text { design trial }\end{array}$ & VLCKD (VLCD) & $\begin{array}{l}61 \text { obese patients } \\
\text { divided in three group: } \\
\text { 1. VLCKD (18) } \\
\text { 2. MetDiet (21) } \\
\text { 3. BS (22) }\end{array}$ & 2 months & $\begin{array}{l}\text { In this study, as in other previous ones, in patients who } \\
\text { underwent VLCKD there was a significant increase in Alistipes } \\
\text { (Rikenellaceae family) while a decrease in Lactobacillus was } \\
\text { recorded. There was also a decrease in Orodibacter } \\
\text { splanchnicus (which in the previous study by the same author } \\
\text { only increased if it was accompanied by probiotic } \\
\text { supplementation) and there was an increase of Parabacteroides. }\end{array}$ \\
\hline $\begin{array}{l}\text { Guitérrez-Repiso, } \\
2019 \text { (23) }\end{array}$ & $\begin{array}{l}\text { Randomized, } \\
\text { single blind, } \\
\text { parallel- } \\
\text { design }\end{array}$ & $\begin{array}{l}\text { VLCKD (VLCD) } \\
75 \mathrm{~g} \text { protein } \\
20 \mathrm{~g} \text { carbohydrates } \\
3 \mathrm{~g} \text { fat }\end{array}$ & $\begin{array}{l}33 \text { obese patients } \\
\text { divided in three group: } \\
\text { 2- synbiotics1- } \\
\text { sunbiotc2, } \\
\text { 3- placebo- } \\
\text { synbiotic2, control. }\end{array}$ & $\begin{array}{l}2 \text { months } \\
\text { (VLCKD) + } \\
2 \text { months } \\
\text { (LCD) }\end{array}$ & $\begin{array}{l}\text { The authors verify that the VLCKD program not alter the gut } \\
\text { microbial population and that the Bacteroidetes/Firmicutes ratio } \\
\text { correlates significantly with the percentage of weight loss. In } \\
\text { particular, it is evident, in the placebo/synbiotc2 group, that the } \\
\text { administration of probiotics such as Bifidobacterium animal } \\
\text { subps lactis and prebiotic fibers was able to increase the weight } \\
\text { gain (compared to the control) also decreasing the } \\
\text { inflammatory state. }\end{array}$ \\
\hline
\end{tabular}

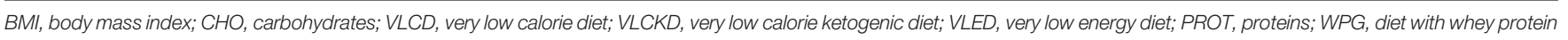
group; VPG, diet with vegetable protein group; APG, diet with animal protein group; MetDiet, Mediterranean Diet, BS, Bariatric Surgery. 
TABLE 2 | Studies on the effects of VLCarbDs on gut microbiota.

\begin{tabular}{|c|c|c|c|c|c|}
\hline AUTHOR, YEAR & TYPE OF STUDY & TYPE OF DIET & SAMPLE & DURATION & FINDINGS \\
\hline $\begin{array}{l}\text { Ley RE at al., } 2006 \\
\text { (25) }\end{array}$ & Randomized trial & VLCarbD (CARB-R) & $\begin{array}{l}12 \text { obese } \\
\text { individuals }\end{array}$ & 52 weeks & $\begin{array}{l}\text { CARB-R diets led to an increase in Bacteroidetes and a decrease in } \\
\text { Firmicutes, increasing the Bacteroidetes: Firmicutes ratio, which was } \\
\text { lower in obese patients than in healthy individuals. }\end{array}$ \\
\hline
\end{tabular}

CARB-R, charbohydrate - restricted; CHO, carbohydrate; HPLC, high-protein/low-carbohydrate; LC, high-protein low carbohydrate ketogenic; SCFAs, short-chain fatty acids; VLCarbD, very low-carbohydrate diet.

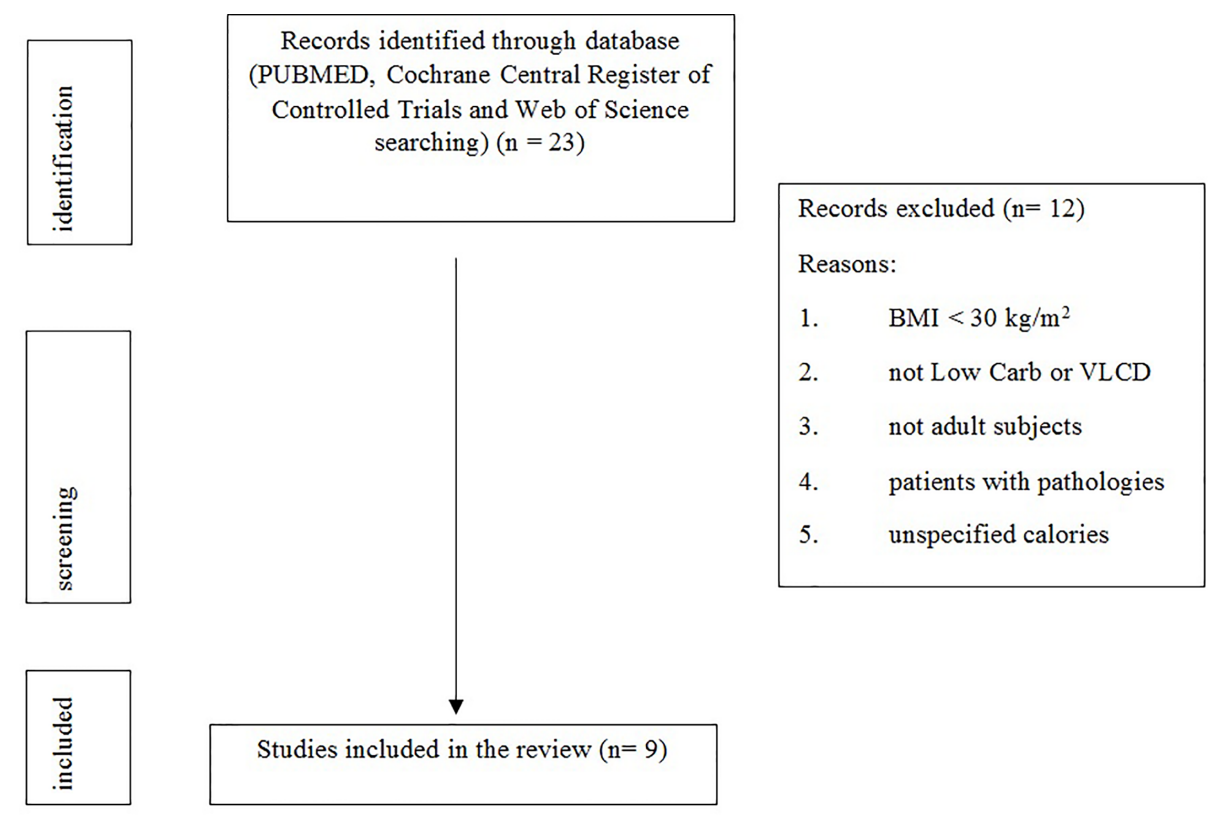

FIGURE 1 | Flow diagram of the review process.

decreased irrespective of diet type as long as the person lost at least $6 \%$ of their body weight $(20,25)$.

VLCarbDs and VLCDs appeared to reduce the abundance of just a few groups of bacteria with health benefits, and they were also associated with increased proportions of others, such as $A$. municiphila and Christensenella. The increased proportions of bacteria, such as Akkermansia and Christensenella, induced by a low-calorie intake, represents an intriguing example of hostmicrobe co-evolution that would appear to benefit both partners $(6,30,31)$. Akkermansia muciniphila is inversely correlated with disease status (32). Akkermansia muciniphila, is a mucin-degrading bacterium that resides in the mucus layer. The presence of this bacterium inversely correlates with body weight in rodents and humans. However, the precise physiological roles played by this bacterium during obesity and metabolic disorders are unknown (33).
Low carbohydrates intake in very low energy diet (VLED) reduced substrates available for Bifidobacteria and bacteria within the Lactobacillus group in the large intestine, while the high content of protein contributed to Bacteroides spp increase (18), the predominant proteolytic species identified in the human large intestine (34). In maintenance phase, at the end of VLED, Bifidobacteria and bacteria within the Lactobacillus group start to increase again suggesting that change is transient. Drastic dietary changes in VLED leads so to Bacteroides spp. increase and to Bifidobacteria decrease, reflecting that bacteria flora modifications are associated with dietary intake rather the body weight variations (18).

Duncan et al. investigated the effects on gut microbiota of two diets, high-protein low carbohydrate, ketogenic (LC) and highprotein moderate-carbohydrate, non-ketogenic (MC). Impact of each diet on gut microbiota was very different: particularly the LC 
diet led to decrease of Firmicutes (Roseburia and Eubacterium rectale) some of which are responsible for butyrate production (26).

Similarly, Russell et al. observed that the populations of the butyrate producing Roseburia/E. rectale group of bacteria decreased markedly with the high protein and low carbohydrate (HPLC) normocaloric diet. The abundance of another group of butyrate producers is maintained, which are related to Faecalibacterium prausnitzii (35). This group may have become the main supplier of butyrate in the intestine with this diet. Butyrate is the main source of energy for the colon cells; therefore, it allows cells to replicate and function normally, avoiding the apoptosis. Faecalibacterium prausnitzii exercises beneficial anti-inflammatory effects on the intestinal mucosa (36) and it is believed to have a positive influence on colon health (35).

The bacteria that are most affected by the decrease in carbohydrate intake are $E$. rectale and Roseburia while $F$. prausnitzii seems to be less affected by the decrease in carbohydrates in the diet. Duncan et al., monitored dietary effect shift from normal intakes of carbohydrate $(399 \mathrm{~g} /$ day) to either moderate $(164 \mathrm{~g} /$ day) or low $(24 \mathrm{~g} /$ day) intakes in a weight loss program for obese individuals, showing a E. rectale and Roseburia spp. marked progressive decrease with reduction of carbohydrate intake (24).

Roseburia and E. rectale belong to the Firmicutes phylum and they are part of the commensal bacteria that produce short chain fatty acids (SCFA), in particular butyrate, which influence colon motility, immunity maintenance and anti-inflammatory properties. Changes in the presence of Roseburia can lead to the development of various diseases (including irritable bowel syndrome, obesity, type 2 diabetes, nervous system conditions and allergies) (37).

Faecalibacterium prausnitzii, an anerobic bacterium belonging to the Firmicutes phylum, is a key component of the gut microbiota and the most abundant butyrate-producing bacterium in the human colon (38). It accounts for approximately 5\% of all detectable bacteria in stool samples from healthy adults (39). Faecalibacterium prausnitzii is best known as a biomarker for human health, as decreased levels have been associated with increased inflammatory activity and may trigger certain diseases such as colorectal cancer (38). Reduced numbers have been reported in inflammatory bowel disease and infectious colitis, and Furet et al. (40) showed that this species might also play a role in low-grade inflammatory disorders such as obesity and diabetes. The authors also observed a relationship between F. prausnitzii and inflammatory markers in both non-diabetic and diabetic obese patients, even after adjustment for BMI. Faecalibacterium prausnitzii proportions were smaller in those patients with type 2 diabetes presenting higher levels of low-grade inflammation and insulin resistance. A negative association was also seen between $F$. prausnitzii and insulin resistance assessed using the homeostatic model assessment for insulin resistance (HOMA IR), possibly attributable to an improvement in glucose metabolism in the diabetic group (40).

In contrast to the previous studies, Aleman et al., observed that during a VLCKD the increased lipolysis leaded to production of $\beta$-hydroxybutyrate ( $\mathrm{BHB})$, a ketone body that is also a short chain fatty acid byproduct, that resulted negatively correlated with F. prausnitzii and genus Roseburia. The increase of BHB levels could results from less catabolism by F. prausnitzii and Roseburia (21).

Further studies on VLCKD were carried out between 2019 and 2021. Guiterrez Repiso et al. revealed that the VLCKD program not alter the gut microbial population and that the Bacteroidetes/ Firmicutes ratio correlates significantly with the percentage of weight loss (23). VLCKD positively changes the microbiota but this change is greater if probiotics and prebiotics are supplemented during the diet (23).

A recent study by Basciani et al. showed that the microbiota is sensitive to the type of proteins of the diet. Patients were administered with a VLCKDs with whey, vegetable or animal proteins; the relative abundance of Firmicutes was significantly decreased while Bacteroidetes increased proportionally with the only exception in the vegetable protein group in which the increase in Bacteroidetes not reached statistical significance suggesting that the origin of proteins may influence the microbiota change (20).

More recently Guiterrez-Repiso et al. compared the effects on the intestinal microbiota in patients following the Mediterranean diet (MetDiet), VLCKD, and who underwent bariatric surgery (BS) (22).

In patients administered with VLCKD there was a significant increase in Alistipes (Rikenellaceae family) while a decrease in Lactobacillus was recorded. There was also a decrease in Orodibacter splanchnicus and there was an increase of Parabacteroides. Alistipes and Parabacteroides were negatively associated with waistline and body mass index in adults (41) and young people (42).

The gut microbiota is a very dynamic entity influenced by nutritional behaviors (43). The composition of the gut microbiota differs in obese and lean subjects, suggesting that microbiota dysbiosis can contribute to changes in body weight (44). While nutritional status (normal weight, overweight) is known to influence the microbial population, there are also studies that have evaluated the influence of the changes in microbiota on the pathways related to obesity and weight loss. In fact, the Firmicutes phylum has been shown to be negatively correlated with the resting energy expenditure (REE) as well as positively correlated with fat mass percentage (45). Moreover, a $20 \%$ increase in the Firmicutes phylum abundance was associated with an increase of $150 \mathrm{kcal}$ in energy harvest (46). On the other hand, a decrease in the Firmicutes-to-Bacteroidetes ratio after a weight loss program also was observed, and the Bacteroidetes proportion was positively correlated with a percentage of loss of body fat (25). In addition, it was observed that the persistent success after a VLCKD (no weight recover in the following 2 years) was associated with the microbiota; the Alistipes, a Bacteroidetes member of Rikenellaceae family was associated with program success, while Prevotella abundance was associated with less success (24).

Several papers investigated the gut microbiota correlation with diet and weight loss, but studies on the effects of specific VLCDs, VLCKDs and VLCarbDs on the microbiota are scarce 
and the results are controversial. The strength of the study is to selectively investigate the available literature on the effect of carbohydrate-restricted diet (VLCDs, VLCKDs and VLCarbDs) on the changes in the intestinal microbiota.

\section{CONCLUSIONS}

Although very few studies have analyzed the effects of VLCarbDs and VLCDs on gut microbiota, significant diet-induced changes

\section{REFERENCES}

1. Devaraj S, Hemarajata P, Versalovic J. The Human Gut Microbiome and Body Metabolism: Implications for Obesity and Diabetes. Clin Chem (2013) 59:617-28. doi: 10.1373/clinchem.2012.187617

2. Clarke G, Stilling RM, Kennedy PJ, Stanton C, Cryan JF, Dinan TG. Minireview: Gut Microbiota: The Neglected Endocrine Organ. Mol Endocrinol (2014) 28:1221-38. doi: 10.1210/me.2014-1108

3. Rastelli M, Knauf C, Cani PD. Gut Microbes and Health: A Focus on the Mechanisms Linking Microbes, Obesity, and Related Disorders. Obes (Silver Spring) (2018) 26:792-800. doi: 10.1002/oby.22175

4. Nicholson JK, Holmes E, Kinross J, Burcelin R, Gibson G, Jia W, et al. HostGut Microbiota Metabolic Interactions. Science (80-) (2012) 336:1262-7. doi: $10.1126 /$ science. 1223813

5. Breban M. Gut Microbiota and Inflammatory Joint Diseases. Jt Bone Spine (2016) 83:645-9. doi: 10.1016/j.jbspin.2016.04.005

6. Goodrich JK, Waters JL, Poole AC, Sutter JL, Koren O, Blekhman R, et al. Human Genetics Shape the Gut Microbiome. Cell (2014) 159:789-99. doi: 10.1016/j.cell.2014.09.053

7. Cheng M, Ning K. Stereotypes About Enterotype: The Old and New Ideas. Genomics Proteomics Bioinforma (2019) 17:4-12. doi: 10.1016/j.gpb.2018.02.004

8. Arumugam M, Raes J, Pelletier E, Le Paslier D, Yamada T, Mende DR, et al. Enterotypes of the Human Gut Microbiome. Nature (2011) 473:174-80. doi: 10.1038/nature09944

9. Larsen N, Vogensen FK, van den Berg FWJ, Nielsen DS, Andreasen AS, Pedersen BK, et al. Gut Microbiota in Human Adults With Type 2 Diabetes Differs From non-Diabetic Adults. PLoS One (2010) 5:e9085. doi: 10.1371/ journal.pone.0009085

10. Cani PD, Possemiers S, Van de Wiele T, Guiot Y, Everard A, Rottier O, et al. Changes in Gut Microbiota Control Inflammation in Obese Mice Through a Mechanism Involving GLP-2-driven Improvement of Gut Permeability. Gut (2009) 58:1091-103. doi: 10.1136/gut.2008.165886

11. Musso G, Gambino R, Cassader M. Obesity, Diabetes, and Gut Microbiota: The Hygiene Hypothesis Expanded? Diabetes Care (2010) 33:2277-84. doi: $10.2337 / \mathrm{dc} 10-0556$

12. Ott B, Skurk T, Hastreiter L, Lagkouvardos I, Fischer S, Büttner I, et al. Effect of Caloric Restriction on Gut Permeability, Inflammation Markers, and Fecal Microbiota in Obese Women. Sci Rep (2017) 7:11955. doi: 10.1038/s41598-017-12109-9

13. Merra G, Gratteri S, De Lorenzo A, Barrucco S, Perrone MA, Avolio E, et al. Effects of Very-Low-Calorie Diet on Body Composition, Metabolic State, and Genes Expression: A Randomized Double-Blind Placebo-Controlled Trial. Eur Rev Med Pharmacol Sci (2017) 21:329-45.

14. Gomez-Arbelaez D, Crujeiras AB, Castro AI, Martinez-Olmos MA, Canton A, Ordoñez-Mayan L, et al. Resting Metabolic Rate of Obese Patients Under Very Low Calorie Ketogenic Diet. Nutr Metab (Lond) (2018) 15:18. doi: 10.1186/ s12986-018-0249-z

15. Basciani S, Costantini D, Contini S, Persichetti A, Watanabe M, Mariani S, et al. Safety and Efficacy of a Multiphase Dietetic Protocol With Meal Replacements Including a Step With Very Low Calorie Diet. Endocrine (2015) 48:863-70. doi: 10.1007/s12020-014-0355-2

16. Tsai AG, Wadden TA. The Evolution of Very-Low-Calorie Diets: An Update and Meta-Analysis. Obes (Silver Spring) (2006) 14:1283-93. doi: 10.1038/oby.2006.146 in fecal microbiota composition have been observed. Further studies are needed.

\section{AUTHOR CONTRIBUTIONS}

MR designed and wrote the paper; CG, GP, SP, PB, IS, RM and $\mathrm{CR}$ wrote and edited the paper, MF and MN visualized the paper. All authors contributed to the article and approved the submitted version.

17. Egger M, Smith GD, Altman DG. Systematic Reviews in Health Care: MetaAnalysis in Context. BMJ Books. BMJ Books Systematic Reviews in Health Care: Meta-Analysis in Context (2001). doi: 10.1002/9780470693926

18. Simões CD, Maukonen J, Scott KP, Virtanen KA, Pietiläinen KH, Saarela M. Impact of a Very Low-Energy Diet on the Fecal Microbiota of Obese Individuals. Eur J Nutr (2014) 53:1421-9. doi: 10.1007/s00394-013-0645-0

19. Louis S, Tappu R-M, Damms-Machado A, Huson DH, Bischoff SC. Characterization of the Gut Microbial Community of Obese Patients Following a Weight-Loss Intervention Using Whole Metagenome Shotgun Sequencing. PLoS One (2016) 11:e0149564. doi: 10.1371/journal.pone.0149564

20. Basciani S, Camajani E, Contini S, Persichetti A, Risi R, Bertoldi L, et al. VeryLow-Calorie Ketogenic Diets With Whey, Vegetable, or Animal Protein in Patients With Obesity: A Randomized Pilot Study. J Clin Endocrinol Metab (2020) 105:1-11. doi: 10.1210/clinem/dgaa336

21. Alemán JO, Bokulich NA, Swann JR, Walker JM, De Rosa JC, Battaglia T, et al. Fecal Microbiota and Bile Acid Interactions With Systemic and Adipose Tissue Metabolism in Diet - Induced Weight Loss of Obese Postmenopausal Women. J Transl Med (2018) 16:1-17. doi: 10.1186/s12967-018-1619-z

22. Gutiérrez-Repiso C, Molina-Vega M, Bernal-López MR, Garrido-Sánchez L, García-Almeida JM, Sajoux I, et al. Different Weight Loss Intervention Approaches Reveal a Lack of a Common Pattern of Gut Microbiota Changes. J Pers Med (2021) 11:109. doi: 10.3390/jpm11020109

23. Gutiérrez-Repiso C, Hernández-García C, García-Almeida JM, Bellido D, Martín-Núñez GM, Sánchez-Alcoholado L, et al. Effect of Synbiotic Supplementation in a Very-Low-Calorie Ketogenic Diet on Weight Loss Achievement and Gut Microbiota: A Randomized Controlled Pilot Study. Mol Nutr Food Res (2019) 63:e1900167. doi: 10.1002/mnfr.201900167

24. Duncan SH, Belenguer A, Holtrop G, Johnstone AM, Flint HJ, Lobley GE. Reduced Dietary Intake of Carbohydrates by Obese Subjects Results in Decreased Concentrations of Butyrate and Butyrate-Producing Bacteria in Feces. Appl Environ Microbiol (2007) 73:1073-8. doi: 10.1128/ AEM.02340-06

25. Ley RE, Turnbaugh PJ, Klein S, Gordon JI. Human Gut Microbes Associated With Obesity. Nature (2006) 444:1022-3. doi: 10.1038/4441022a

26. Duncan SH, Lobley GE, Holtrop G, Ince J, Johnstone AM, Louis P, et al. Human Colonic Microbiota Associated With Diet, Obesity and Weight Loss. Int J Obes (2008) 32:1720-4. doi: 10.1038/ijo.2008.155

27. Qin J, Li R, Raes J, Arumugam M, Burgdorf KS, Manichanh C, et al. A Human Gut Microbial Gene Catalogue Established by Metagenomic Sequencing. Nature (2010) 464:59-65. doi: 10.1038/nature08821

28. Ley RE, Hamady M, Lozupone C, Turnbaugh PJ, Ramey RR, Bircher JS, et al. Evolution of Mammals and Their Gut Microbes. Science (80-) (2008) 320:1647-51. doi: 10.1126/science.1155725

29. Reddel S, Putignani L, Del Chierico F. The Impact of Low-Fodmaps, Glutenfree, and Ketogenic Diets on Gut Microbiota Modulation in Pathological Conditions. Nutrients (2019) 11:373. doi: 10.3390/nu11020373

30. Dao MC, Everard A, Aron-Wisnewsky J, Sokolovska N, Prifti E, Verger EO, et al. Akkermansia Muciniphila and Improved Metabolic Health During a Dietary Intervention in Obesity: Relationship With Gut Microbiome Richness and Ecology. Gut (2016) 65:426-36. doi: 10.1136/gutjnl-2014-308778

31. Davenport ER, Sanders JG, Song SJ, Amato KR, Clark AG, Knight R. The Human Microbiome in Evolution. BMC Biol (2017) 15:1-12. doi: 10.1186/s12915-017-0454-7 
32. Geerlings SY, Kostopoulos I, De Vos WM, Belzer C. Akkermansia Muciniphila in the Human Gastrointestinal Tract : When, Where, and How? Microorganisms (2018) 6:1-26. doi: 10.3390/microorganisms6030075

33. Shin N, Lee J, Lee H, Kim M, Whon TW, Lee M, et al. An Increase in the Akkermansia Spp. Population Induced by Metformin Treatment Improves Glucose Homeostasis in Diet-Induced Obese Mice. Gut (2014) 63:727-35. doi: 10.1136/gutjnl-2012-303839

34. Macfarlane GT, Cummings JH, Allison C. Protein Degradation by Human Intestinal Bacteria. J Gen Microbiol (1986) 132:1647-56. doi: 10.1099/ 00221287-132-6-1647

35. Russell WR, Gratz SW, Duncan SH, Holtrop G, Ince J, Scobbie L, et al. HighProtein, Reduced-Carbohydrate Weight-Loss Diets Promote Metabolite Profiles Likely to be Detrimental to Colonic Health. Am J Clin Nutr (2011) 93:1062-72. doi: 10.3945/ajen.110.002188

36. Zhao L, Zhang F, Ding X, Wu G, Lam YY, Wang X, et al. Gut Bacteria Selectively Promoted by Dietary Fibers Alleviate Type 2 Diabetes. Science (2018) 359:1151-6. doi: 10.1126/science.aao5774

37. Tamanai-Shacoori Z, Smida I, Bousarghin L, Loreal O, Meuric V1, Fong SB, et al. Roseburia Spp.: A Marker of Health? Future Microbiol (2017) 12:157-70. doi: $10.2217 / \mathrm{fmb}-2016-0130$

38. Ferreira-Halder CV, Faria AVS, Andrade SS. Action and Function of Faecalibacterium Prausnitzii in Health and Disease. Best Pract Res Clin Gastroenterol (2017) 31:643-8. doi: 10.1016/j.bpg.2017.09.011

39. Louis P, Young P, Holtrop G, Flint HJ. Diversity of Human Colonic ButyrateProducing Bacteria Revealed by Analysis of the Butyryl-Coa:Acetate CoA-transferase Gene. Environ Microbiol (2010) 12:304-14. doi: 10.1111/j.1462-2920.2009.02066.x

40. Furet J-P, Kong L-C, Tap J, Poitou C, Basdevant A, Bouillot J-L, et al. Differential Adaptation of Human Gut Microbiota to Bariatric SurgeryInduced Weight Loss: Links With Metabolic and Low-Grade Inflammation Markers. Diabetes (2010) 59:3049-57. doi: 10.2337/db10-0253

41. Zeng Q, Li D, He Y, Li Y, Yang Z, Zhao X, et al. Discrepant Gut Microbiota Markers for the Classification of Obesity-Related Metabolic Abnormalities. Sci Rep (2019) 9:13424. doi: 10.1038/s41598-019-49462-w
42. Lv Y, Qin X, Jia H, Chen S, Sun W, Wang X. The Association Between Gut Microbiota Composition and BMI in Chinese Male College Students, as Analysed by Next-Generation Sequencing. Br J Nutr (2019) 122:986-95. doi: 10.1017/S0007114519001909

43. Paoli A, Mancin L, Bianco A, Thomas E, Mota JF, Piccini F. Ketogenic Diet and Microbiota: Friends or Enemies? Genes (Basel) ((2019) 10:534. doi: 10.3390/genes10070534

44. Cuevas-Sierra A, Ramos-Lopez O, Riezu-Boj JI, Milagro FI, Martinez JA. Diet, Gut Microbiota, and Obesity: Links With Host Genetics and Epigenetics and Potential Applications. Adv Nutr (2019) 10(suppl_1):S17-30. doi: 10.1093/ advances/nmy078

45. Kocełak P, Zak-Gołąb A, Zahorska-Markiewicz B, Aptekorz M, Zientara M, Martirosian G, et al. And Gut Microbiota in Obese and Normal Weight Subjects. Eur Rev Med Pharmacol Sci (2013) 17:2816-21. doi: 10.1155/2013/674106

46. Jumpertz R, Le DS, Turnbaugh PJ, Trinidad C, Bogardus C, Gordon JI, et al. Energy-Balance Studies Reveal Associations Between Gut Microbes, Caloric Load, and Nutrient Absorption in Humans. Am J Clin Nutr (2011) 94:58-65. doi: 10.3945/ajen.110.010132

Conflict of Interest: PB, IS and RM were employed by company PronoKal Group.

The remaining authors declare that the research was conducted in the absence of any commercial or financial relationships that could be construed as a potential conflict of interest.

Copyright (C) 2021 Rondanelli, Gasparri, Peroni, Faliva, Naso, Perna, Bazire, Sajoux, Maugeri and Rigon. This is an open-access article distributed under the terms of the Creative Commons Attribution License (CC BY). The use, distribution or reproduction in other forums is permitted, provided the original author(s) and the copyright owner(s) are credited and that the original publication in this journal is cited, in accordance with accepted academic practice. No use, distribution or reproduction is permitted which does not comply with these terms. 